\title{
Estudio comparativo y aplicaciones de algoritmos de filtrado adaptable
}

\author{
L.H. Escobar-Salguero \\ Departamento de Electrónica, División de Ingeniería Eléctrica \\ Facultad de Ingeniería, UNAM \\ E-mail: larrye@ servidor.unam.mx
}

(recibido: enero de 2003; aceptado: junio de 2003)

\begin{abstract}
Resumen
El filtrado adaptable está ganando espacio en campos tales como procesamiento de voz, sistemas de comunicaciones y redes de alta velocidad, en donde los parámetros varían en el tiempo. Existen muchos algoritmos de filtrado adaptable que pueden ser usados para resolver problemas en áreas como comunicaciones, control e instrumentación. Este trabajo presenta los algoritmos de filtrado adaptable (AFA) más utilizados, sus principales rasgos y ventajas, con el fin de mostrar sus desempeños para seleccionar el más adecuado. También se muestran algunas aplicaciones de filtrado adapt able que son difíciles de realizar con filtrado fijo.
\end{abstract}

Descriptores: filtrado adapt able, algoritmos de filtrado adapt able, predicción lineal, cancelación de ruido, cancelación de eco, igualación de canal.

\begin{abstract}
A dap tivefil ter ing is gain ing spacein many fields such as voiceprocessing, com mu ni ca tionssystems and high speed net works wherethepa ram eters aretime varying. Therearea lot of adap tivefiltering al go rithms (AFA) that wecan useto solveprob lemsin com mu ni ca tions, con trol and in stru $\mathrm{m}$ en ta tion. This work pres entsthemost com mon adap tivefil ter ing al go rithms (AFA), their ma jor features and thead van tages in or der to showtheir per for manceand choosethebest. Alsoweshow someap plica tions of adap tivefil tering (AF) that are diffic cult to carry out us ing a fixed fil ter in $\mathrm{g}$.
\end{abstract}

Keywords: adaptivefil tering, adaptivefil ter ingal gorithms, lin ear prediction, noisecancel la tion, echocan cel lation, chan nel equal ization.

\section{Introducción}

En la actualidad, el filtrado adaptable se ha convertido en una herramienta de utilidad para una gran cantidad de aplicaciones, principalmente en áreas como control, instrumentación y telecomunicaciones.
La propiedad principal de un sistema adaptable es la de autoajustar su desempeño, es decir, si se aplica una señal a la entrada el sistema se adapta en relación a los cambios que presenta la misma, esto significa que es difícil caracterizar un sistema adaptable en términos convencionales. En general, un 
sistema adaptable es aquel que se adecúa a los cambios del medio en donde interactúa para efectuar correctamente su desempeño (Widrow y Stearns, 1985). En el procesamiento adaptable de señales existe un número finito de parámetros que son ajustados por los algoritmos al optimizar un criterio de desempeño (Sibul, 1987 y Haykin, 1991).

Entre los problemas donde se pueden aplicar los AFA se tiene como ejemplo un canal de comunicaciones digitales que atenuará, retrasará y distorsionará la señal que se propaga a través de éste, es decir, que afectará las características de las señales que viajan a través del canal y que porta los símbolos digitales a transmitir. Para eliminar estos efectos nocivos en la señal transmitida, se requiere tener filtros de alto desempeño para cancelar ruido, así como procesos que sean capaces de cancelar el eco de la señal y eliminar la interferencia intersimbólica (ISI) (Haykin, 1991 y Proakis, 1995).

En el área de control, existen procesos donde es necesario identificar una planta desconocida, de tal forma que la función de transferencia de la misma pueda ser especificada. Los sistemas adaptables de identificación se utilizan para modelar las variaciones lentas de una planta cuyas entradas y salidas son disponibles, tal es el caso de estudios de vibraciones mecánicas (Escobar, 1997 y Haykin, 1991); (Widrow y Stearns, 1985).

En el área de instrumentación es muy frecuente el proceso de filtrar una señal que está contaminada por ruido, particularmente en instrumentación biomédica para una señal de EGC, uno de los efectos nocivos es la interferencia de $60 \mathrm{hz}$ de la alimentación de corriente alterna, que además de interferir puede moverse de su frecuencia de $60 \mathrm{hz}$ y afectar el intervalo de frecuencias de la señal de ECG. Para cancelar la interferencia de $60 \mathrm{hz}$ el uso de filtros fijos no es muy adecuado. Entre las técnicas más utilizadas para cancelar estas interferencias se encuentra el filtrado adaptable, que no requiere de un conocimiento a pri ori de la función de transferencia del canal ni de los estadísticos de las señales a transmitir (Proakis, 1995).

En este trabajo se presenta el desempeño de los AFA y los campos de aplicación, entre los que se encuentra la predicción lineal, la cancelación de interferencias tales como la cancelación de ruido en varias señales y la cancelación de una señal de $60 \mathrm{hz}$ en una señal de ECG, la cancelación de eco que es un efecto nocivo en las comunicaciones y la igualación de canal en comunicaciones digitales que ha sido un problema muy estudiado y todavía permanece vigente en la actualidad. Se presentan las formas de implantación de los algoritmos para las aplicaciones y se muestran los resultados obtenidos.

Asimismo, se analizan los sistemas adaptables y los algoritmos AFA, las aplicaciones generales de los algoritmos AFAs, los algoritmos AFAs, el desempeño de estos algoritmos, los resultados obtenidos por el autor en las diferentes aplicaciones, así como la discusión y conclusiones de los resultados.

\section{Estructura de los algoritmos AFAs}

En esencia, un sistema adaptable es variable en el tiempo y autoajusta su desempeño. Por otro lado, en un sistema fijo, el diseñador tiene un panorama completo de las posibles entradas, sus estadísticas y conoce qué debe hacerse bajo cada una de estas circunstancias. 
El diseñador tendrá que elegir un criterio específico para juzgar la cantidad de error entre el sistema real y el modelo seleccionado del sistema.

Cuando se desconocen las condiciones de entrada y sus estadísticas o las condiciones cambian en el tiempo, los sistemas adaptables buscan constantemente el desempeño óptimo dentro de todas las posibilidades permitidas.

La estructura de un sistema adaptable se muestra en la figura 1, para una señal de entrada $x(n)$ y una señal de respuesta deseada $d(n)$ (n es el tiempo discreto), se tiene una señal de error e(n) que es la diferencia entre la señal deseada $d(n)$ y la señal de salida $y(n)$.

La salida y(n) se estima con base en la señal de entrada $x(n)$ y los parámetros actuales del bloque de proceso, en el sumador se calcula el error

$$
e(n)=d(n)-y(n)
$$

de un criterio de desempeño estima los parámetros óptimos del proceso de tal forma que se minimice el error estimado.

Dependiendo de la aplicación particular el esquema de la figura 1 puede cambiar ( Haykin, 1991 y Proakis, 1995); (Widrow y Stearns, 1985).

La mayoría de los AFAs minimizan la suma del error medio cuadrático, es decir:

$$
E=\sum_{n=0}^{M} e^{2}(n)=\sum_{n=0}^{M}\left[d(n)-\sum_{i=0}^{N-1} c(i) x(n-i)\right]^{2}
$$

Para hallar el mínimo de la ecuación (2) con respecto a los coeficientes $c(n)$ del filtro se deberesolver

donde $d(n)$ es una señal deseada que depende del proceso a diseñar, el error se alimenta al bloque del algoritmo adaptable, que a través

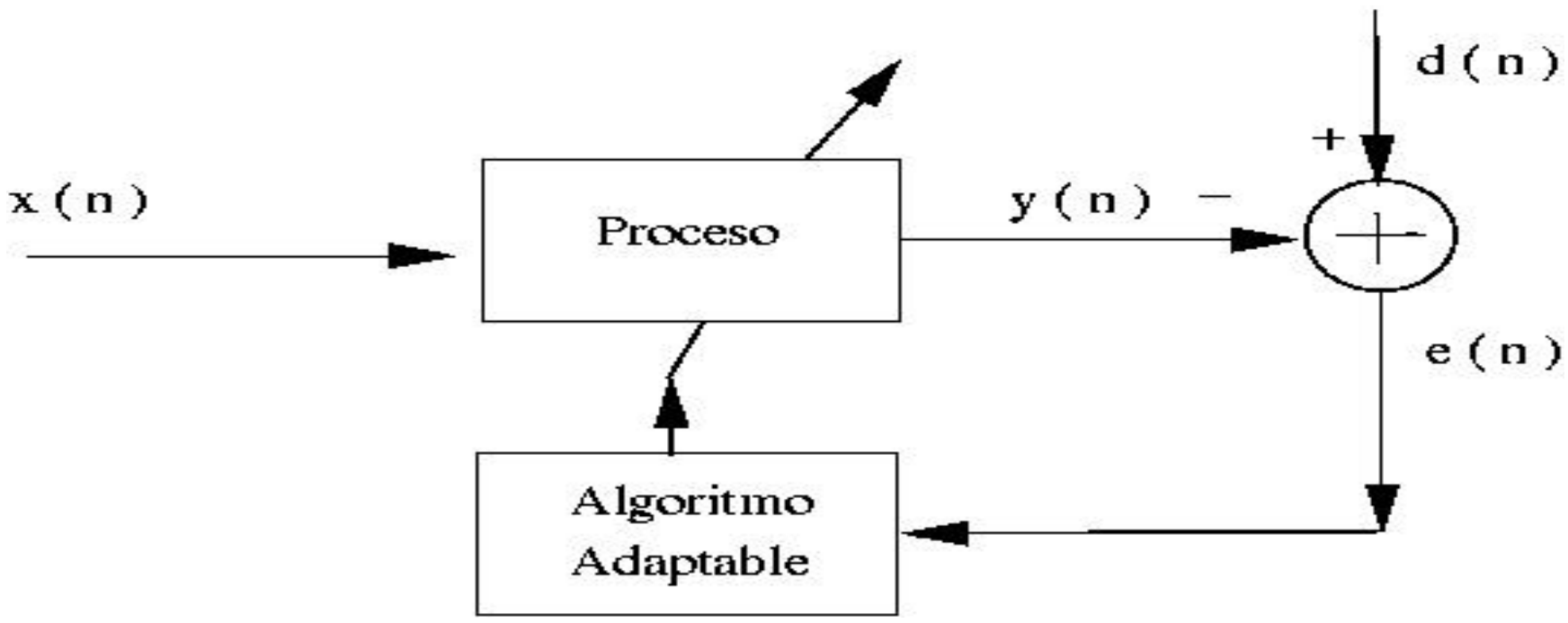

Figura 1. Estructura de un sistema adaptable 
que conduce a la solución de Wiener-Hopf (Alcántara, 1986; Haykin, 1991; Proakis, 1995 y Widrow, 1985) ${ }^{1}$

$$
C_{p}(n)=R_{p}^{-1}(n-1) r_{p}(n)
$$

\section{Sistemas adaptables}

La operación de un sistema adaptable involucra dos procesos básicos, el filtrado y la adaptación (Figura 1):

1. El proceso de filtrado está diseñado para producir una respuesta 0 secuencia de salida para una secuencia de entrada dada.

2. El proceso adaptable provee un mecanismo para el control de un conjunto de parámetros de ajuste usados en el proceso de filtrado.

\section{Aplicaciones de los AFAs}

La diferencia esencial entre las diversas aplicaciones de filtrado adapt able es la manera en que la respuesta deseada es excitada. Bajo esta idea es posible configurar diferentes estructuras de sistemas adaptables que se explican a continuación y se muestran en la figura 2. (Haykin, 1991); (Widrow y Stearns, 1985).

\section{Predicción}

Tal vez es la aplicación más simple, los retrasos de la señal de entrada $x(n)$ son enviados al proceso adaptable (Figura 2.a), éste debe tratar de predecir la entrada actual de la señal $x(n)$ para minimizar el error

1 Los desarrollos matemáticos se pueden encontrar en la bibliografía mencionada cuadrático. La predicción es utilizada en la codificación o compresión de señales, procesamiento de voz, codificación diferencial DPCM, codificación adaptable ADPCM, análisis espectral, radar, sonar, procesos geológicos, sismología, etc. (Haykin, 1989; Proakis, 1995; Widrow y Stearns, 1985; Haykin, 1991 y Alcántara, 1986).

\section{Identificación de sistemas}

En este caso la señal transmitida $x(n)$ es la de entrada al proceso adaptable y a una planta desconocida (Figura 2.b). Después de la adaptación, la planta es identificada en el sentido que la función de transferencia de la misma queda especificada como la del proceso adaptable. Otra aplicación de interés es la cancelación de eco en líneas telefónicas.

\section{M odelo inverso o deconvolución}

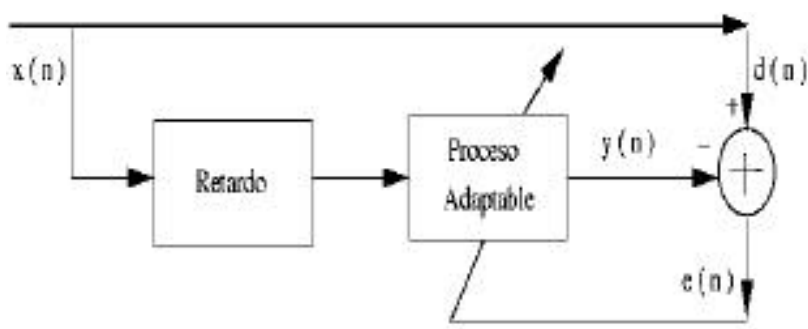

a) PREDICCION

Figura 2a. A plicaciones de los algoritmos AFA

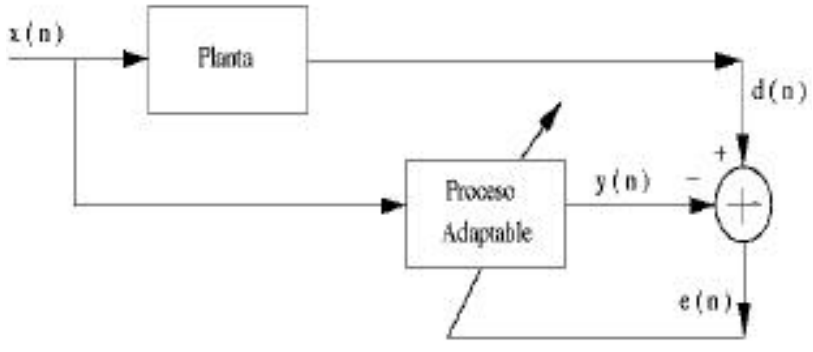

b) IDENTIFICACION DE SISTEMAS

Figura 2b. A plicaciones de los algoritmos A F A 
El proceso adaptable intenta recuperar una versión retardada de la señal de entrada $x(n)$, esta señal retardada sería la señal deseada, la cual se supone que ha sido alterada por las variaciones lentas de la planta y el contenido de ruido (Figura 2.c). La igualación adaptable es utilizada para la deconvolución de los efectos de un transductor, un canal de comunicación o la producción de un modelo inverso de una planta. También es aplicable en el diseño de filtros digitales (Alcántara, 1986; Haykin, 1991,1994 y Widrow y Stearns, 1985).

Cancelación de ruido

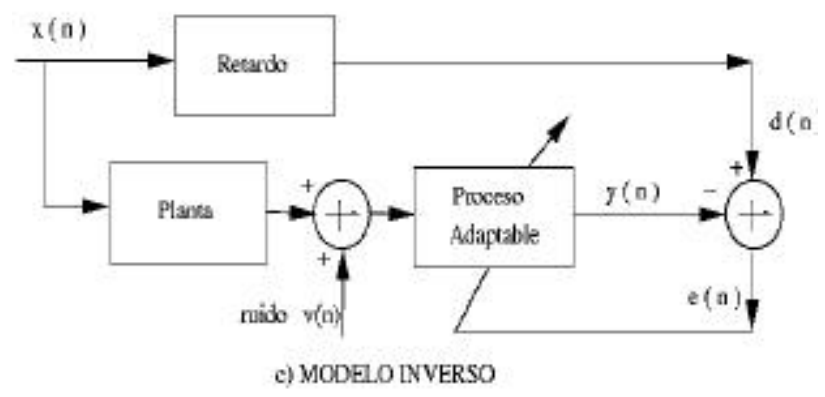

Figura 2c. A plicaciones de los algoritmos AFA

En este caso la señal $x(n)$ está corrompida por ruido $v(n)$, y se dispone de una versión correlacionada de ruido $v_{1}(n)$ que entra al proceso adaptable (Figura 2.d). El objetivo del proceso adaptable es producir una salida y(n) similar a $v(n)$. Es decir, minimizar el error cuadrático de e(n) (Alcántara, 1986; Haykin, 1991 y Proakis, 1995). La señal e(n) sería la señal deseada de salida y debe ser aproximadamente la señal $x(n)$ sin ruido.

\section{Algoritmos de filtrado adaptable}

Un algoritmo AFA efectúa una minimización de la señal de error e(n) utilizando un criterio particular, el criterio más utilizado es el que minimiza el error cuadrático. Si $N$ es el orden de un filtro transversal se tienen los siguientes algo ritmos.

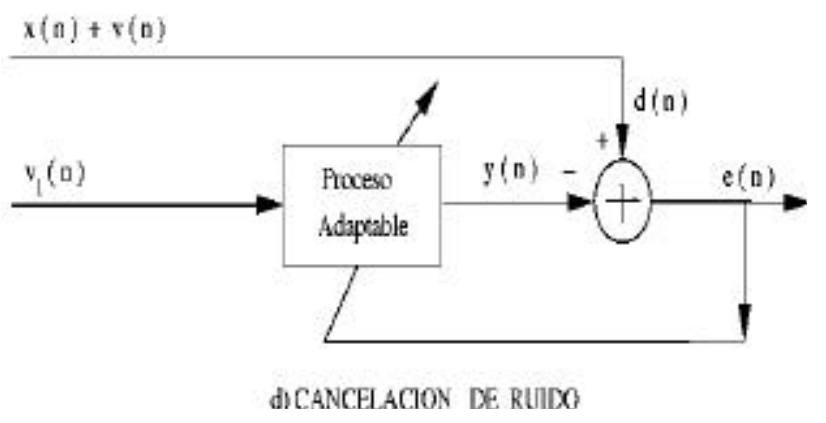

Figura $2 \mathrm{~d}$. A plicaciones de los algoritmos A FA

\section{Algoritmo LM S}

Este algoritmo hace una minimización sobre promedios temporales del error cuadrático medio (MSE) (Widrow y Stearns, 1985), se le llama aproximado porque hace un cálculo cercano de los coeficientes $C(n)$. La constante $\mu$ se conoce como tamaño del paso. Este algoritmo evita la inversión de la matriz de autocorrelación de la señal de entrada $\mathbf{R}$ y consta de un proceso adaptable, el cual involucra el reajuste del vec tor de coeficientes y un proceso de filtrado en el que se estima la respuesta deseada. Este algoritmo no requiere del conocimiento de los estadísticos de la señal de entrada al filtro, ya que los promedios probabilísticos requeridos por el algoritmo de los pasos descendentes son sustituidos por los promedios temporales. En este caso, el gradiente $y$ los coeficientes tienen un comportamiento ruidoso, lo que trae como consecuencia que el algoritmo LMS no converja a un valor óptimo en la solución de la ecuación de Wiener-Hopf (Ecuación 4). El algoritmo LMS es un algoritmo tipo gradiente y converge a la solución óptima lentamente, es convergente en la media, es convergente en el 
valor cuadrático medio y exhibe desajuste en estado estable. En la tabla 1 se resumen las ecuaciones del algoritmo LMS.

\section{Tabla 1: Algoritmo LMS}

\begin{tabular}{c}
\hline Inicialización \\
$C_{N}(0)=0$ \\
\hline Ecuaciones \\
\hline$y(n)=C_{N}^{H}(n) x(n)$ \\
$e(n)=d(n)-y(n)$ \\
$C_{N}(n)=C_{N}(n-1)+\mu x(n) e^{*}(n)$ \\
\hline
\end{tabular}

El algoritmo estima la salida y(n) del proceso adaptable, calcula el error e(n) entre la señal deseada d(n) y la señal estimada, y con base a este error, actualiza los coeficientes $C(n)$.

M étodo de mínimos cuadrados (LS)

Este método involucra el uso de promedios en el tiempo, de acuerdo a este método se minimiza un índice de desempeño que consiste en la suma de los errores cuadrados, el error re sid ual es definido como la diferencia entre la respuesta deseada y la respuesta de salida actual del filtro (Haykin, 1991). La solución es similar a solución de Wiener-Hopf (Alcántara, 1986 y Escobar, 1997). Este algoritmo hace una estimación sobre una ventana de datos recibidos de longitud $\mathrm{N}$ y minimiza la sumas de errores al cuadrado respecto de los coeficientes $C_{N}(n)$

$$
\sum_{k=1}^{n}\left\{d(n)-C_{N}^{T}(n-1) x_{N}(n)\right\}^{2}
$$

es decir, obtener la derivada del error cuadrático respecto de los coeficientes $C_{\mathrm{N}}(\mathrm{n}) \mathrm{e}$ igualarla a cero, conduce a la solución conocida de Wiener-Hopf. La solución de los
LS se realiza en una ventana de datos, por lo que la solución es global, y es necesario calcular el inverso de la matriz $\mathbf{R}$ para obtener la solución, este proceso implica efectuar operaciones del orden $\mathrm{N}^{3}$.

\section{Algoritmo RLS}

El algoritmo RLS parte de la solución global del algoritmo LS. La deducción de este algoritmo se basa en el lema de inversión matricial y estima los coeficientes $C(n)$ de un filtro transversal, por cada muestra se hace una actualización de los coeficientes en el tiempo $\mathrm{n}$ tomando en cuenta las $N$ entradas anteriores (Sibul, 1987 y Haykin, 1991).

Los coeficientes $C_{N}(n)$ se pueden actualizar recursivamente

$$
C_{N}(n)=C_{N}(n-1)+K_{N}(n) e(n)
$$

donde

$$
K_{N}(n) \equiv R_{N}^{-1}(n) x_{N}(n)
$$

es el vector de ganancia $K_{N}(n)$ conocido como Ganancia de Kalman y se puede generar recursivamente sin necesidad de estar invirtiendo la matriz $R_{N}^{-1}(n)$.

Un criterio de desempeño que considera los cambios del sistema es la minimización del error cuadrático en el tiempo $n$ multiplicado por un fac tor de peso exponencial $\lambda$, conocido como factor de olvido, el cual tiene un valor muy cercano a uno. Al inverso de $1-\lambda$ se le conoce como la memoria del algoritmo Entonces la matriz $R_{N}(n)$ se puede calcular recursivamente como 


$$
R_{N}(n)=\lambda R_{N}(n-1)+x_{N}(n) x_{N}(n)^{T}
$$

Los coeficientes se siguen calculando recursivamente como en la ecuación (6). En la tabla 2 se resumen las ecuaciones del algoritmo RLS.

Los desarrollos de las ecuaciones se pueden encontrar en (Alcántara, 1986; Escobar, 1997 y Haykin, 1991). Las operaciones efectuadas por el algoritmo RLS son $4 N^{2}+4 N$ multiplicaciones y $3 N^{2}+N-1$ sumas. La literatura reporta que la convergencia del algoritmo RLS es del orden $2 \mathrm{~N}$ iteraciones cuando la media del error de convergencia es pequeña comparado con la señal deseada d(n) (Haykin, 1991).

Tabla 2: Algoritmo RLS

\begin{tabular}{c} 
Inicialización \\
$C_{N}(0)=W_{N}(0)=K_{N}(0),=0, P_{N}(0)=\delta I_{N}$ \\
$\delta=$ Cte. positiva pequeña \\
I= Matriz unitaria \\
\hline$e(n)=d(n)+C_{T}^{N}(n-1) X_{N}(n-1)$ \\
$C_{N}(n)=C_{N}(n-1)+K_{N}(n-1) e(n)$ \\
$W_{N}(n)=P_{N}(n-1) X_{N}(n)$ \\
$\gamma_{N}(n) \frac{1}{1-W_{N}^{T}(n) X_{N}(n)}$ \\
$\mathrm{K}_{N}(n)=\gamma_{N}(n) W_{N}(n)$ \\
$P_{N}(n)=P_{N}(n-1)-K_{N}(n) W_{N}^{T}(n)$
\end{tabular}

\section{Algoritmos rápidos}

El algoritmo RLS tiene una complejidad computacional que se incrementa con el cuadrado de $N$, donde $N$ es el número de coeficientes ajustables de filtro adaptable. Tales algoritmos son frecuentemente referidos como algoritmos $O\left(N^{2}\right)$, donde $O($.) denota "orden de" 0 número de operaciones aritméticas, (Carayannis et al., 1983 y Alcántara, 1986). Un algoritmo rápido tiene como propósito explotar la estructura de la matriz de autocorrelación de los datos para reducir el número de operaciones a una cantidad $O(N)$. Entre los algoritmos rápidos se pueden identificar los siguientes:

Algoritmo RLS rápido (FRLS) o Algoritmo rápido de Kalman (ARK)

Como su nombre lo indica, es un algoritmo que proviene del RLS, éste explota la redundancia en el algoritmo LS y para su realización efectúa la predicción hacia adelante y hacia atrás (Carayannis, 1983), (Falconer y Ljung, 1978). Su nombre proviene de un vector de ganancia similar a la aproximación del filtrado de Kalman para la formulación en vari ables de estado.

El algoritmo FRLS hace una estimación hacia adelante y una estimación hacia atrás de la señal de entrada al proceso adaptable, de esta forma, se calcula un vector de ganancia $K_{N}(n)$ de orden $N+1$ conocido como ganancia de Kalman, este algoritmo explota al máximo las relaciones que se derivan del lema de inversión matricial permitiendo llegar a expresiones más simples para su implantación (Alcántara, 1986; Falconer y Ljung, 1978; Haykin, 1991; Proakis, 1992 y Sibul, 1987). Este algoritmo simplifica el tipo de operaciones, sólo realiza operaciones como productos escalares, escalar por escalar, escalar por vec tor, suma de vectores y división entre escalares.

La solución del problema queda expresada como un conjunto de recursiones de una matriz actualizada en el tiempo. El algoritmo FRLS puede tratar con ambientes estacionarios y no estacionarios, su limitación básica es su complejidad de cálculo de coeficientes matriciales. El vector $C(n)$ es un 
estimador hacia adelante y el vector $B(n)$ es un estimador hacia atrás (Alcántara, 1986; Falconer y Ljung, 1978; Haykin, 1991; Proakis, 1992 y Sibul, 1987). El número de operaciones de este algoritmo es del orden $8 \mathrm{~N}$ por iteración.

\section{A lgoritmo de filtrado transversal rápido (FTF)}

Este algoritmo reemplaza algunas operaciones vectoriales del algoritmo FRLS por operaciones escalares, reduciendo el número de operaciones respecto del ARK (Cioffi, 1984 y Haykin, 1991). El algoritmo FTF se basa en la combinación de cuatro filtros transversales separados trabajando en conjunto (Cioffi, 1984 y Haykin, 1991):

- El predictor de error hacia adelante.

- El predictor de error hacia atrás.

- Cálculo de la ganancia de algoritmo RLS.

- Filtro de parámetros cuyo vector de parámetros es la estimación deseada.

La ventaja de este algoritmo respecto al FRLS es que efectúa $5 \mathrm{~N}$ operaciones por iteración.

\section{A goritmo recursivo de los mínimos cuadrados Lattice (LSL)}

Utiliza estructuras "lattice", para efectuar el filtrado y la actualización de sus parámetros se basa en la estimación de los mínimos cuadrados de los predictores hacia adelante y hacia atrás.

Algoritmos lattice de mínimos cuadrados

Basados en la descomposición matricial QR, en este algoritmo las formas de predicción hacia adelante y hacia atrás son explotadas con el propósito de reducir la complejidad computacional en la ejecución de la descomposición $Q R$ de la matriz de datos de entrada.

\section{Comparación y desempeño}

En este punto se hace una comparación de los algoritmos tratados en este trabajo. En la figura 4 se muestra una comparación de la convergencia del error cuadrático para varios algoritmos. Es evidente que para los algo ritmos de tipo gradiente, tal como el LMS, su velocidad de convergencia es muy lenta, por lo que para aplicaciones donde se requieren respuestas muy rápidas este algoritmo no es conveniente, ya que cuando se hace crecer la constante $\mu$ el algoritmo tiende a converger más rápido; sin embargo, presenta problemas de inestabilidad. En muchas aplicaciones lo que se hace es proponer constantes $\mu$ grandes y luego disminuirlas cuando el algoritmo ha convergido (Escobar, 1997).

En la figura 3 se observa que los algoritmos rápidos desarrollados a partir del algoritmo RLS tienen una razón de convergencia muy rápida, de ahí toman su nombre de rápidos, cuya convergencia del error cuadrático es aproximadamente $2 \mathrm{~N}$ iteraciones. Otra ventaja de los algoritmos RLS es que pueden operar adecuadamente en ambientes no estacionarios. Además, los algoritmos tipo LS y RLS son insensibles a las propiedades de correlación de los datos de entrada en contraste con los algoritmos tipo gradiente, el algoritmo LMS es muy sen si ble a la dispersión de los valores característicos de la matriz de autocorrelación R (Carayannis et al., 1983; Widrow y Stearns, 1985 y Haykin, 1991). 
L.H. Escobar-Salguero

En la tabla 3 se hace un resumen de los algoritmos desarrollados y se compara la cantidad de operaciones teóricas por iteración

Comparación de errores

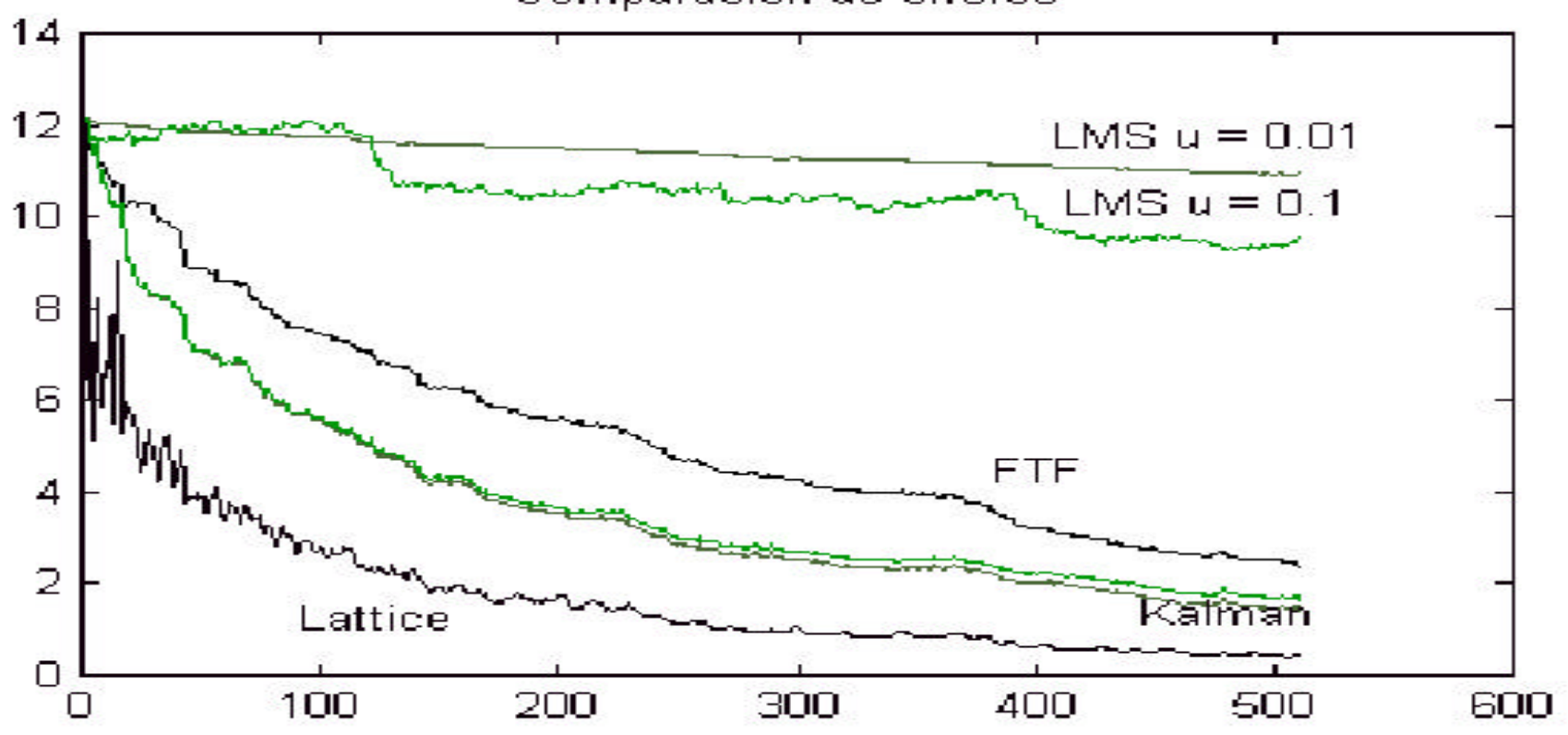

Figura 3. Convergencia del error en AFAs

Original
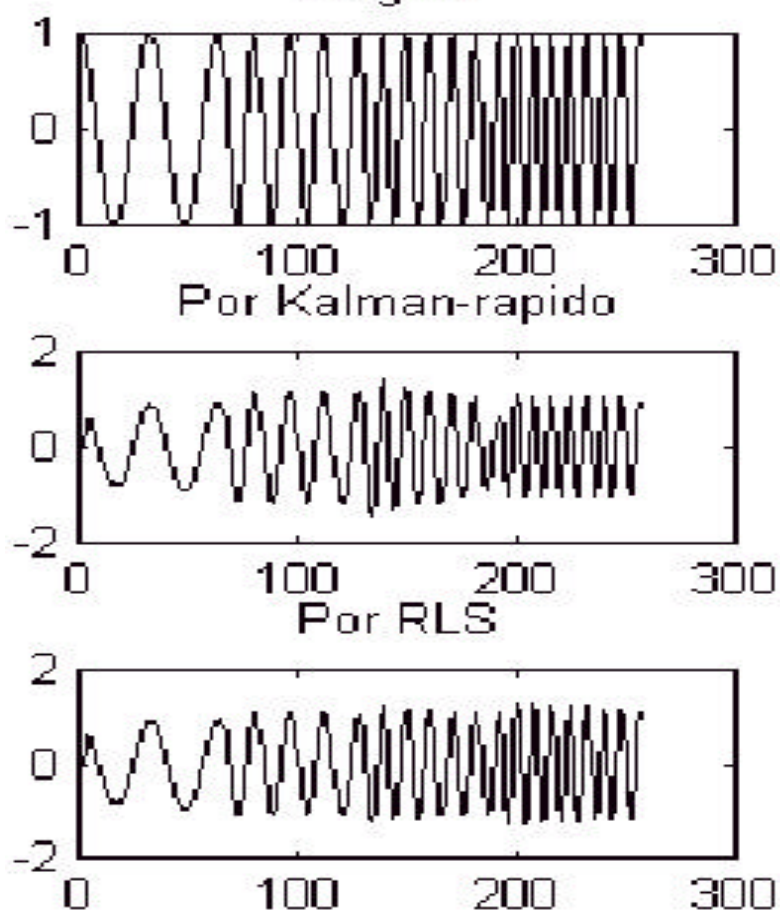

Espectras
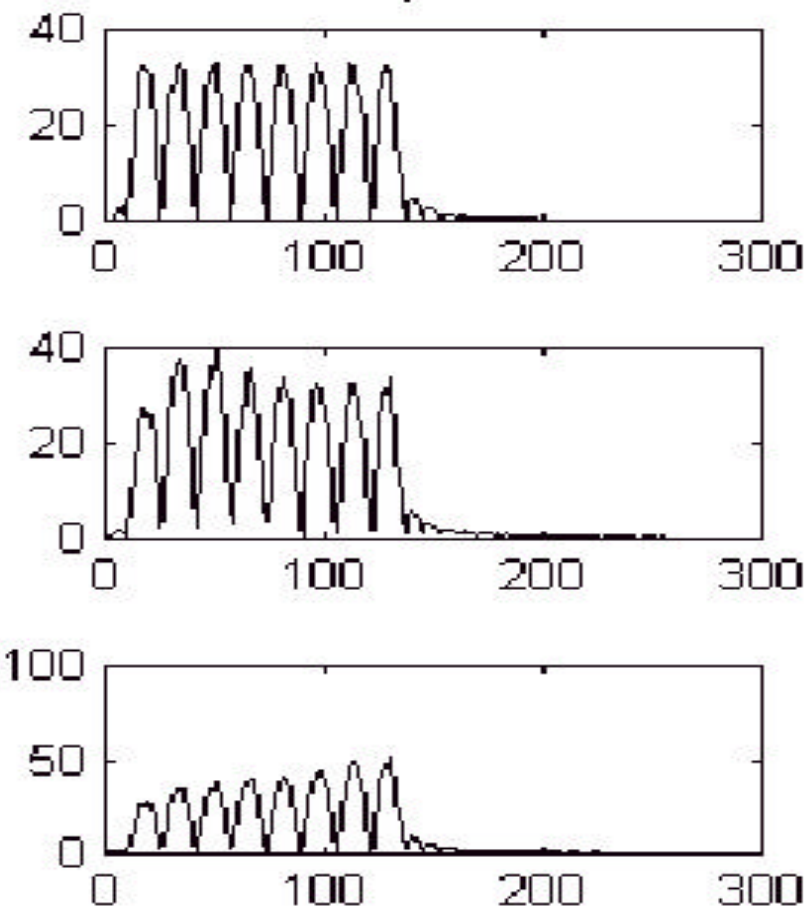

Figura 4. Predicción de una señal cosenoidal variable 
Estudio comparativo y aplicaciones de algoritmos de filtrado adapt able

Tabla 3. Número de operaciones teóricas de AFAs

\begin{tabular}{llcll}
\hline & & Predicción & Filtrado \\
\hline Algoritmo & $*$ & $\div$ & $*$ & $\div$ \\
\hline LMS & $2 \mathrm{~N} \mathrm{a} \mathrm{3N}$ & 1 & $3 \mathrm{~N}$ & 1 \\
RLS & $4 \mathrm{~N}^{2}+4 \mathrm{~N}$ & 1 & & \\
FRLS & $8 \mathrm{~N}$ & 2 & $10 \mathrm{~N}$ & 2 \\
FAEST & $5 \mathrm{~N}+8$ & 2 & $7 \mathrm{~N}+9$ & 2 \\
FTF & $5 \mathrm{~N}$ & 2 & $7 \mathrm{~N}$ & 2 \\
LSALE & $19(\mathrm{~N}+1)-12$ & & & \\
\hline
\end{tabular}

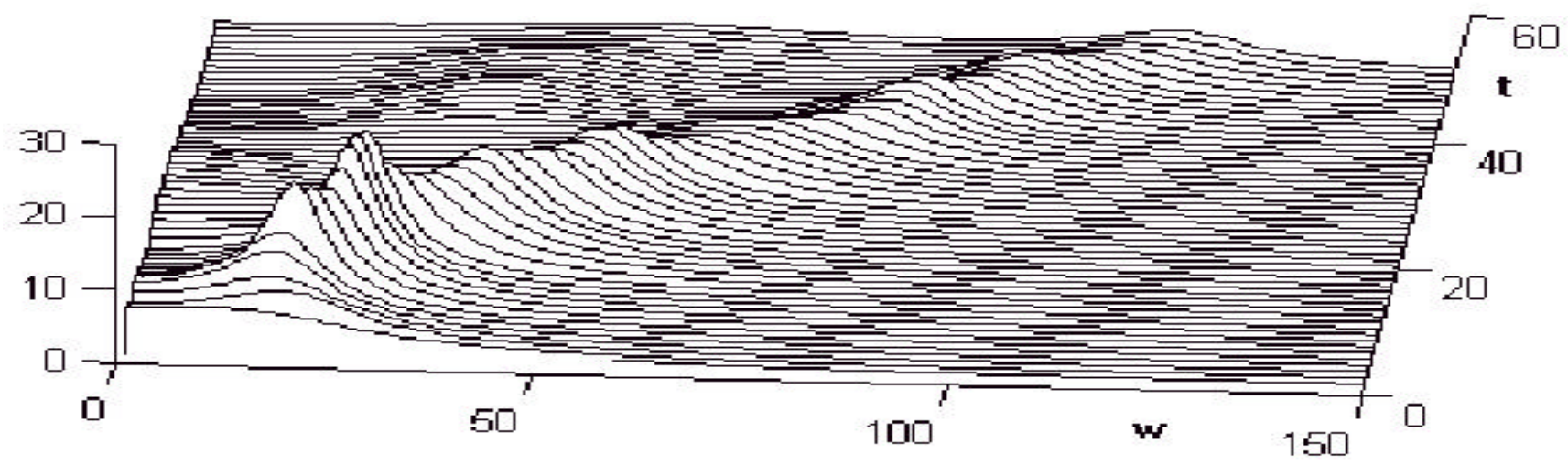

Figura 5. Respuesta espectral del seguimiento de la señal cosenoidal variable

que efectúa cada algo ritmo (multiplicaciones y divisiones). De esta tabla se observa que el algoritmo LMS es el que realiza menos operaciones por iteración; sin em bargo, como ya se mencionó tiene el inconveniente de que su velocidad de convergencia es muy lenta. Los algoritmos rápidos RLS son los que presentan menos cantidad de operaciones por iteración, es decir, $\mathrm{O}(\mathrm{N})$ con convergencia rápida. Algunas pruebas adicionales se pueden encontrar en (Escobar, 1997), donde además se tienen pruebas realizadas en arquitecturas de procesadores de señales digitales (DSP) (Alcántara y Escobar, 1999).

\section{Resultado de las aplicaciones}

Aquí se presentan los resultados de la aplicación de los algoritmos AFA a aplicaciones concretas.

\section{Predicción de señales}

A manera de ejemplo se tiene una señal cosenoidal de frecuencia variable, como se observa en la figura 4. Como se discutió anteriormente, en la medida que el error converge, el algoritmo tiende a la solución óptima y la señal estimada es similar a la señal deseada, casi a partir de la muestra 30. Además, en la columna derecha de la figura 4 se presentan los espectros respectivos.

En la figura 5 se observa la respuesta en frecuencia del algoritmo adaptable, es decir, 
que en la medida que la señal cosenoidal de entrada cambia, el algoritmo modifica la respuesta del sistema para seguir los cambios de la señal, en el eje $\omega$ de frecuencia se observa como el algoritmo va cambiando su respuesta en frecuencia ante una señal cosenoidal.

\section{Cancelación de ruido utilizando AFA}

En esta parte se utilizan los AFAs antes mencionados en la cancelación de ruido en señales. Se realizó una prueba con una señal triangular (Figura 6) con ruido agregado, en este caso, la riqueza espectral de la señal tri angu lar es muy amplia, por lo que la cancelación de ruido se hace más difícil, sobre todo porque se ha disminuido la relación señal a ruido.

\section{Aplicación a una señal de ECG}

La señal de ECG presenta una forma característica cuyas magnitudes y duraciones reflejan la activación cíclica y sincronizada de los millones de células responsables de la contracción del músculo cardiaco o miocardio. La prueba de ECG es un diagnóstico rutinario para diversas enfermedades del corazón, la identificación de ritmos cardiacos irregulares (arritmias), por lo que es necesario contar con una señal libre de interferencias. En la figura 7 se muestra una señal de electrocardiograma (ECG) afectada por la interferencia de una señal senoidal de $60 \mathrm{hz}$. Como se observa en la señal ECG contaminada, existen zonas donde la interferencia es tan grande que la señal real es imperceptible para efectos de dictamen cardiológico.

El ancho de banda de una señal de ECG está entre 0.05 y $100 \mathrm{hz}$; sin embargo, la mayor densidad de potencia espectral se encuentra en el intervalo de 0.3 a $30 \mathrm{hz}$, concentrándose en el complejo QRS (picos de la señal ECG) localizado entre los 2 y $20 \mathrm{hz}$ y teniendo un máximo en 12 hz. Esto no quiere decir que se descarten las componentes espectrales alrededor de $60 \mathrm{hz}$. A la señal de ECG contaminada se le aplicó el mismo esquema de cancelación de ruido para eliminar la señal de $60 \mathrm{hz}$. En la señal filtrada se observa cómo el proceso de filtrado adaptable logra eliminar el efecto de interferencia.

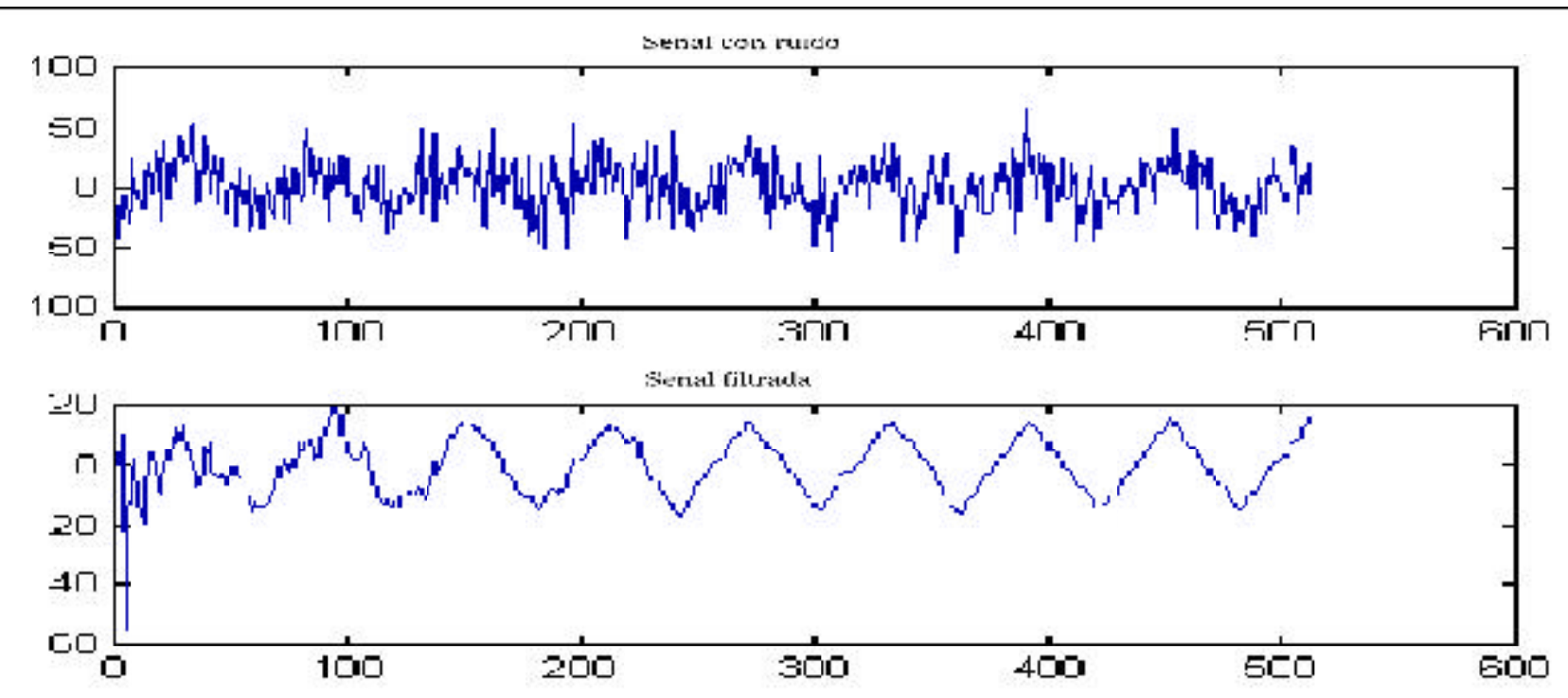

Figura 6. Cancelación de ruido en una señal triangular 


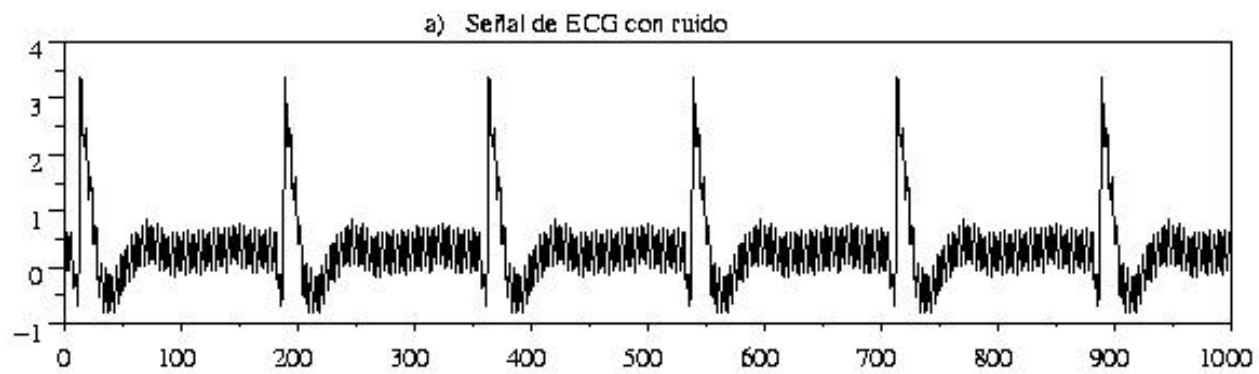

b) Seflal de ECG filtradacon AEA LIMS

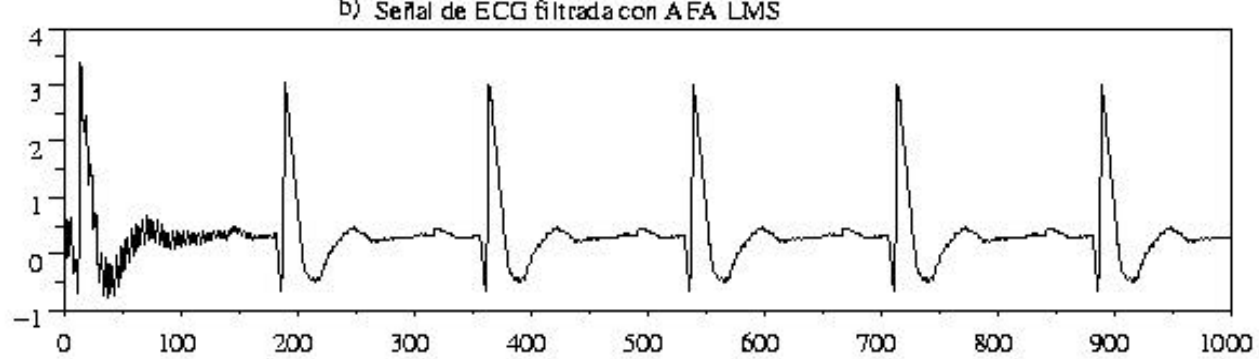

c) Sellal de ECG filtradacon AFA ERLS

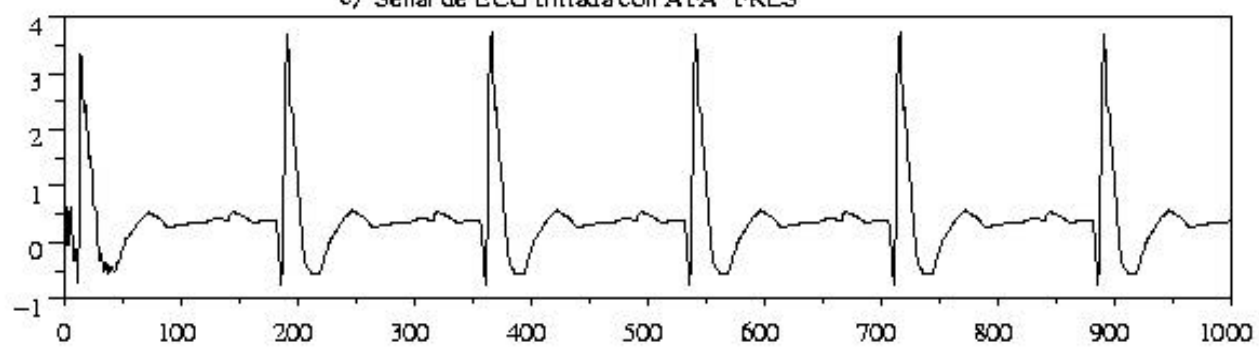

Figura 7. Cancelación de una señal de 60 hz. En una de ECG

señal filtrada siempre se presentan los efectos de la adaptación hasta que el algoritmo adaptable converge. En la figura 7.b se puede observar el resultado de haber utilizado el algoritmo LMS, que converge muy lentamente y en la figura 7.c, se observa el mismo resultado al aplicar un algoritmo adaptable rápido tipo FRLS, en el último caso se puede ver que la convergencia es más rápida. Para una mayor discusión de esta diferencia en los algo ritmos AFAs ver (Alcántara, 1986; Escobar, 1997; Haykin, 1991; Proakis y Manolakis, 1992; Sibul, 1987 y Widrow y Stearns, 1985).
El eco en redes telefónicas es una interferencia nociva que puede hacer indistinguible la información. Una fuente de eco en una red telefónica puede ser una simple conexión entre dos usuarios (abonados o conversadores). Una conexión típica de una simple red telefónica consiste de dos alambres del lado de cada abonado que se conectan a un dispositivo híbrido, dentro de cada dispositivo híbrido existen cuatro ca bles hacia la red gen eral, donde cada par de cables efectúa la transferencia en un sentido (Haykin, 1991 y Messerschmitt et al., 1989). 
Los dispositivos híbridos se encargan de convertir la conexión de dos a cuatro alambres y distribuir el sentido de la transmisión, además debe evitar el retorno de señal a la fuente de emisión, de lo contrario esta sería una fuente de eco. El circuito de cuatro alambres puede usar cables de microondas, fibra óptica y enlaces de microondas. Desafortunadamente, debido al acoplamiento de impedancias de los circuitos híbridos, una parte de la energía de la señal transmitida se retorna, es decir, la per sona que habla percibe una versión retrasada y distorsionada de su propia voz, lo que constituye el eco.

En este trabajo se han hecho pruebas de cancelación de eco bajo el esquema del anulador de eco en la figura 8, y en la figura 9 se muestran las pruebas realizadas para el eco de una señal $y(n)$ que se agrega a una señal senoidal $x(n)$. En la figura 9 se tiene la eliminación de eco utilizando el algo ritmo LMS con $\mu=0.005$ y 100 coeficientes, y para el algo ritmo FRLS se utilizó $\lambda=0.985 \operatorname{con} N=10$.

En la figura 8 se supone que el conversador 1 emite una señal $y(n)$ de pulsos, el conver- sador 2 emite una señal senoidal $x(n)$ que se va a contaminar con el eco de la señal $y(n)$, el error e(n) será la resta de esta señal contaminada, menos el eco estimado por el sistema adapt able.

$$
e(n)=x(n)+r(n)-r^{\prime}(n)
$$

donde un filtro transversal hace la estimación del eco

$$
r^{\prime}(n)=\sum_{i=9}^{p-1} h^{\prime}(i) y(n-i)
$$

$h^{\prime}(n)$ es la respuesta al impulso estimada del canal a través del AFA.

$r^{\prime}(n)$ es la interferencia estimada por el filtro adaptable.

$r(n) \quad$ es la interferencia que gen era el circuito híbrido del escucha 2 y lo retorna al conversador 1 .

e(n) es la señal deseada en el escucha 1 y enviada por el conversador 2 e(n) es minimizada por el criterio de desempeño utilizado por el AFA.

\section{Conversador 1 y $(n)$}

Escucha 2

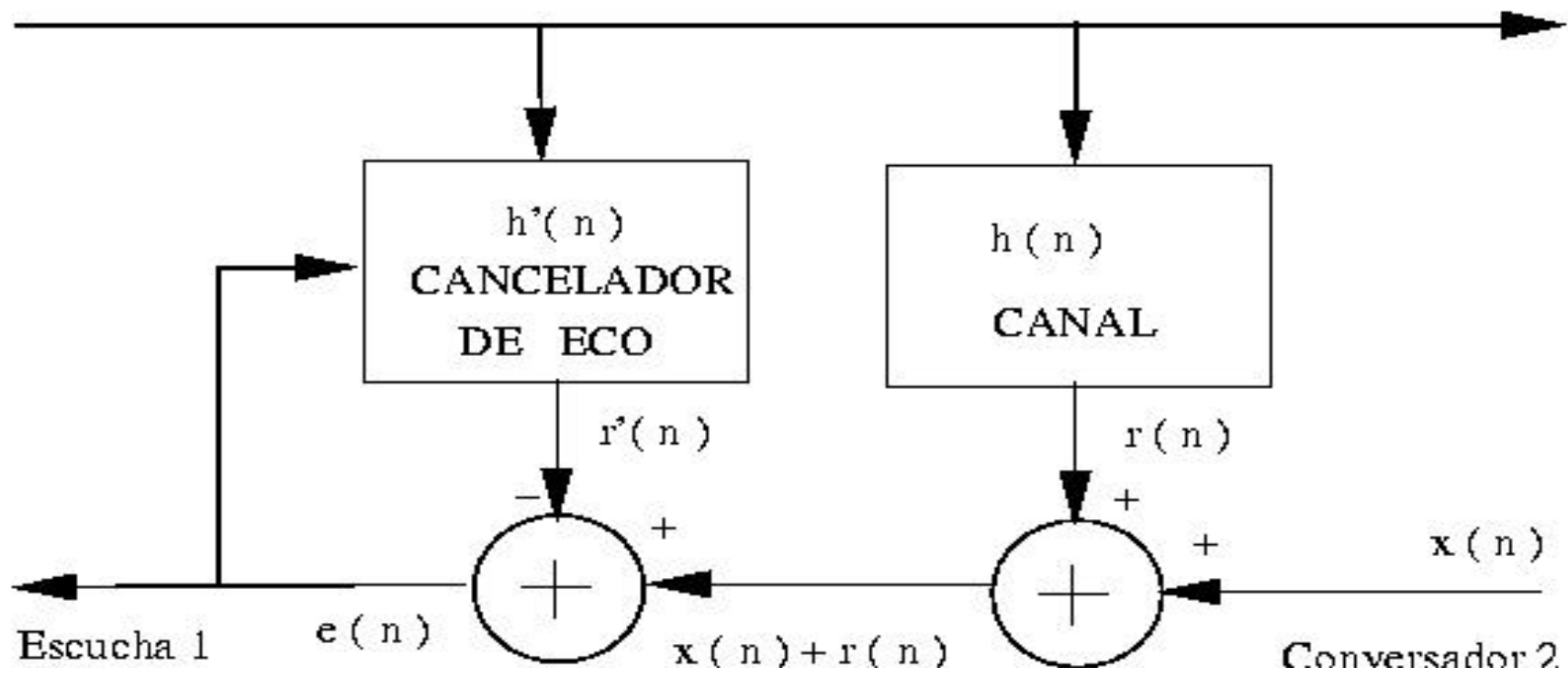

Figura 8. Esquema de cancelación de eco 


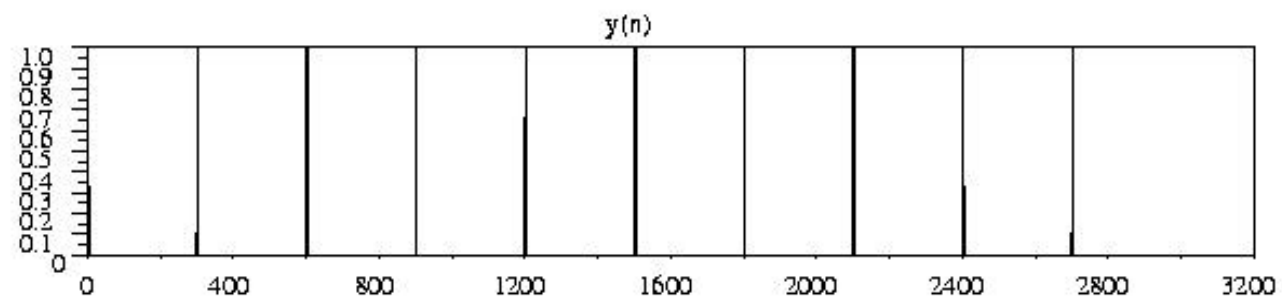

$r(n)$ Eco de seflal $y(n)$
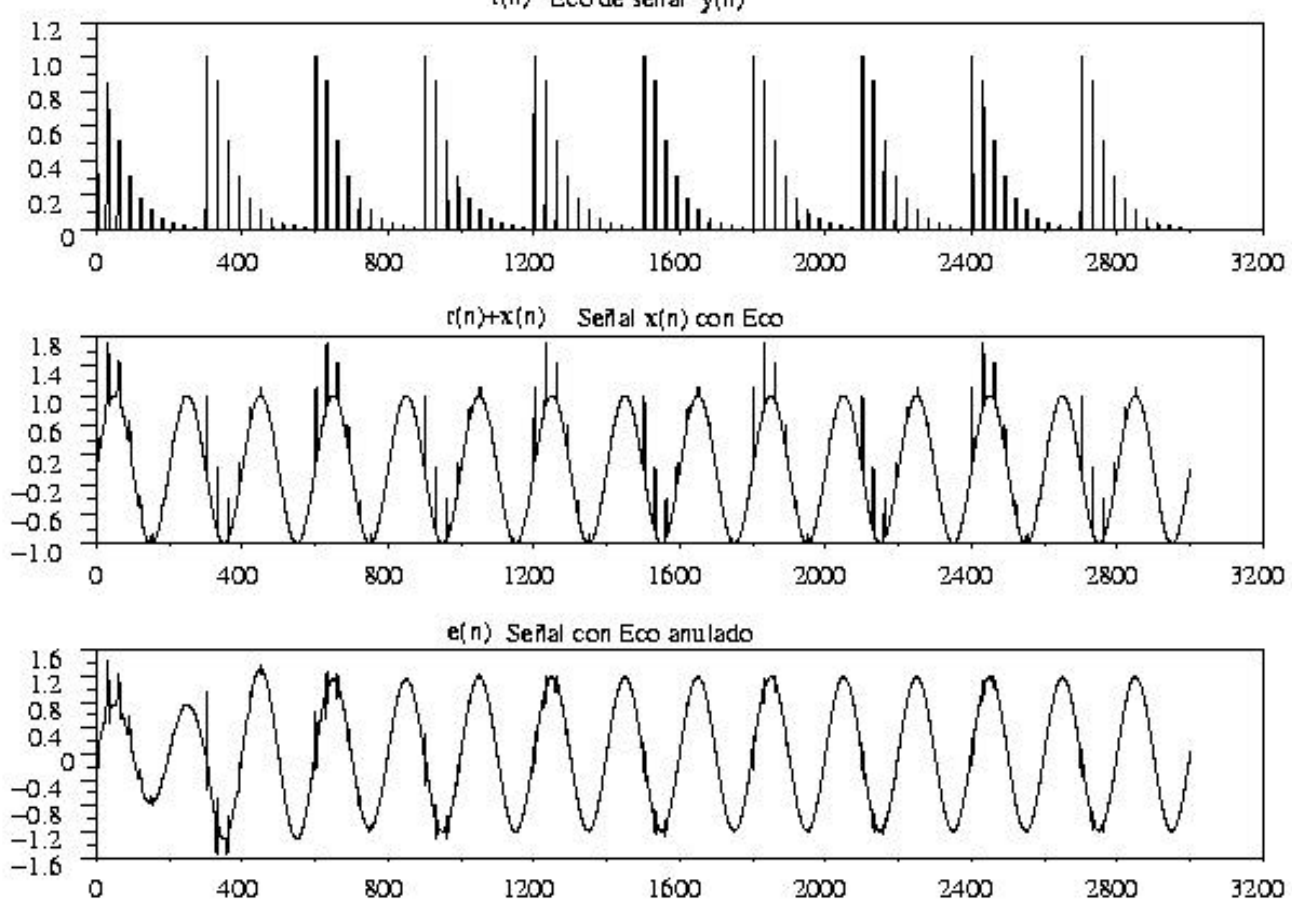

Figura 9. Resultados de la cancelación de eco

En la figura 9 se muestran las pruebas realizadas para una señal $y(n)$, el eco que se agrega a una señal senoidal $x(n)$. En la figura 9 se observa una secuencia de señales utilizadas para la cancelación de eco, en la última señal se observa como se ha logrado eliminar el eco ocasionado por un tren de pulsos de la señal fuente del conversador 1 al pasar por el canal.

\section{Igualación de canal IC}

El problema fundamental para transmitir grandes cantidades de información en tiempo real a través de un canal de comunicaciones digitales, son las restricciones en ancho de banda. En el caso de contar con un ancho de banda infinito se podrían transmitir grandes tasas de información; sin em bargo, ésta es una condición ideal, por lo que es necesario la búsqueda de técnicas para poder transmitir en tiempo real tasas superiores a las que permite el canal. Por ejemplo, en un canal digital la señal de salida presenta una distorsión respecto a la señal original a causa de la interferencia intersimbólica (ISI), esto ocasiona errores cuando se intentan recuperar los datos.

Para resolver este problema es necesario diseñar un sistema correctivo en cascada con 
el sistema original (canal), para producir una réplica de la señal originalmente transmitida, a este sistema se le llama igualador de canal. El objetivo del igualador de canal es remover la distorsión introducida por el canal sobre la señal transmitida.

La idea básica de un igualador de canal se puede pensar como un sistema inverso, porque debe tener una respuesta en frecuencia recíproca a la respuesta en frecuencia del sistema que causó la distorsión, es decir, si el sistema original pro duce una salida r(n) que es la convolución de la entrada $x(n)$ con la respuesta al impulso del canal $h(n)$, entonces la operación de tomar $r(n)$ y obtener $x^{\prime}(n)$ como el valor estimado de $x(n)$ se le llama deconvolución (Proakis y Manolakis, 1992) (Figura 10). La deconvolución es una operación lin eal que remueve el efecto de una convolución previa sobre una secuencia de datos (Haykin, 1991).

Tipos de Igualadores de canal

1. Igualador inverso, la idea de un igualador es que dado un canal con una función de transferencia $H(z)$ entonces la función de transferencia de un igualador de canal en el receptor sería:

$$
G(z)=H^{-1}(z)
$$

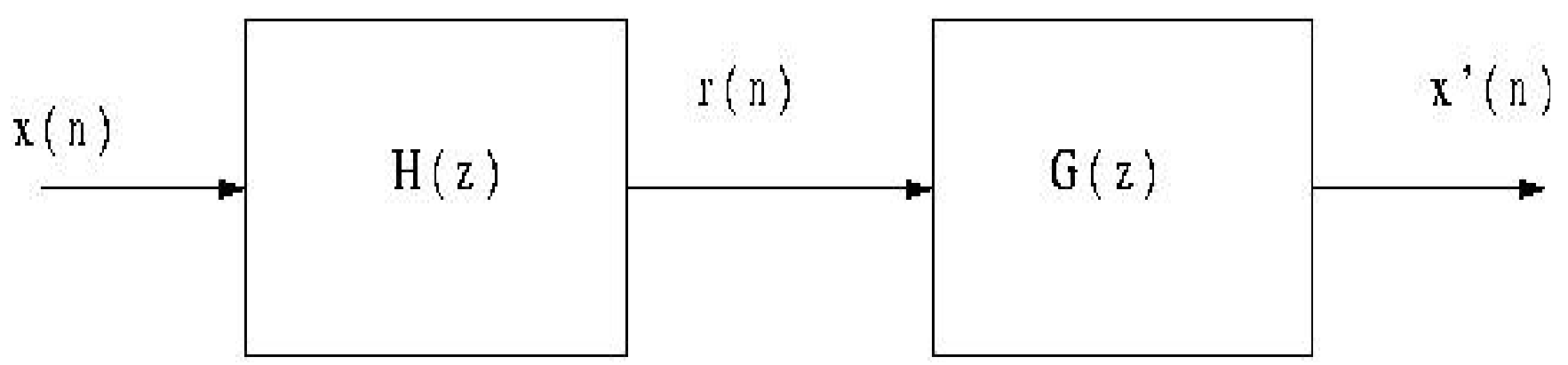

Figura 10. Igualador inverso
Entonces, la función de transferencia total del sistema es el producto entre las funciones $\mathrm{H}(\mathrm{z})$ y $G(z)$

$$
G(z) H(z)=1
$$

cuya transformada $\mathrm{Z}$ inversa es la función $\delta(\mathrm{n})$ Por otro lado, en el tiempo discreto en el receptor

$$
\begin{gathered}
r(n)=x(n) * h(n) \\
x^{\prime}(n)=r(n) * g(n)=x(n) * h(n) * g(n)
\end{gathered}
$$

Pero $h(n) * g(n)$ debería ser igual a $\delta(n)$, ya que $G(z)$ es función inversa de $H(z)$, entonces $x^{\prime}(n)=$ $x(n) * \delta(n)$, es decir, que se tiene la señal deseada en el re cep tor y el ancho de banda se ha convertido infinito. Esta idea no siempre es realizable, ya que depende de la posibilidad de conocer la función de transferencia del canal al invertir la función $\mathrm{H}(\mathrm{z})$, además el canal puede variar en el tiempo.

2. Igualación automática, involucra el envío de una serie de pulsos aislados de prueba previo a la transmisión. Este es un período inicial de entrenamiento que fija los valores del igualador para el período de transmisión de datos. Periódicamente se pueden introducir intervalos cortos de entrenamiento para actualizar los coeficientes del igualador. 
También se le conoce como igualador prefijado.

3. Igualación adaptable, aquí el igualador se estima directamente de la señal recibida, este igualador busca continuamente minimizar las muestras de la señal de salida, con respecto a una señal de referencia que se parece a las ampli tudes de los pulsos transmitidos. Du rante el período inicial de entrenamiento se puede disponer de una señal ideal de referencia en el igualador por medio de la transmisión sobre el canal de una secuencia conocida de datos seudoaleatorios (Figura 11).

Una vez que la distorsión re sid ual decrece a su valor mínimo, los datos verdaderos pueden ser transmitidos. El igualador puede utilizarse en la reconstrucción de la señal recibida como una señal de referencia, esto es llamado Modo de decisión directo. El igualador adaptable tiene ventajas sobre el igualador prefijado, es decir, que se adapta a los cambios de las características del canal durante la transmisión. Existe claramente un retardo en la transmisión de datos que es proporcional a la longitud del período de retardo. En la figura 11 se tiene un diagrama básico de un igualador adaptable con una secuencia de entrenamiento, cuando el igualador está conectado a la secuencia de entrenamiento, el igualador se entrena hasta que el algoritmo converge, entonces, el igua- lador se conecta a la salida (operación en modo directo). Este tipo de igualadores deben seguir los cambios del canal de comu- nicaciones y los algoritmos utilizados deben satisfacer los requerimientos básicos discu- tidos anteriormente (Treichler et al., 1998 y Alcántara, 1986)

El autor ha hecho pruebas utilizando AFAs en la solución del problema de igualación de canal para un canal con una severa ISI (Proakis, 1995 y Alcántara, 1986), donde

$$
H(z)=0.251+0.935 z^{-1}+0.251 z^{-2}
$$

es decir, $h(n)=\{0.251,0.935,0.251$.

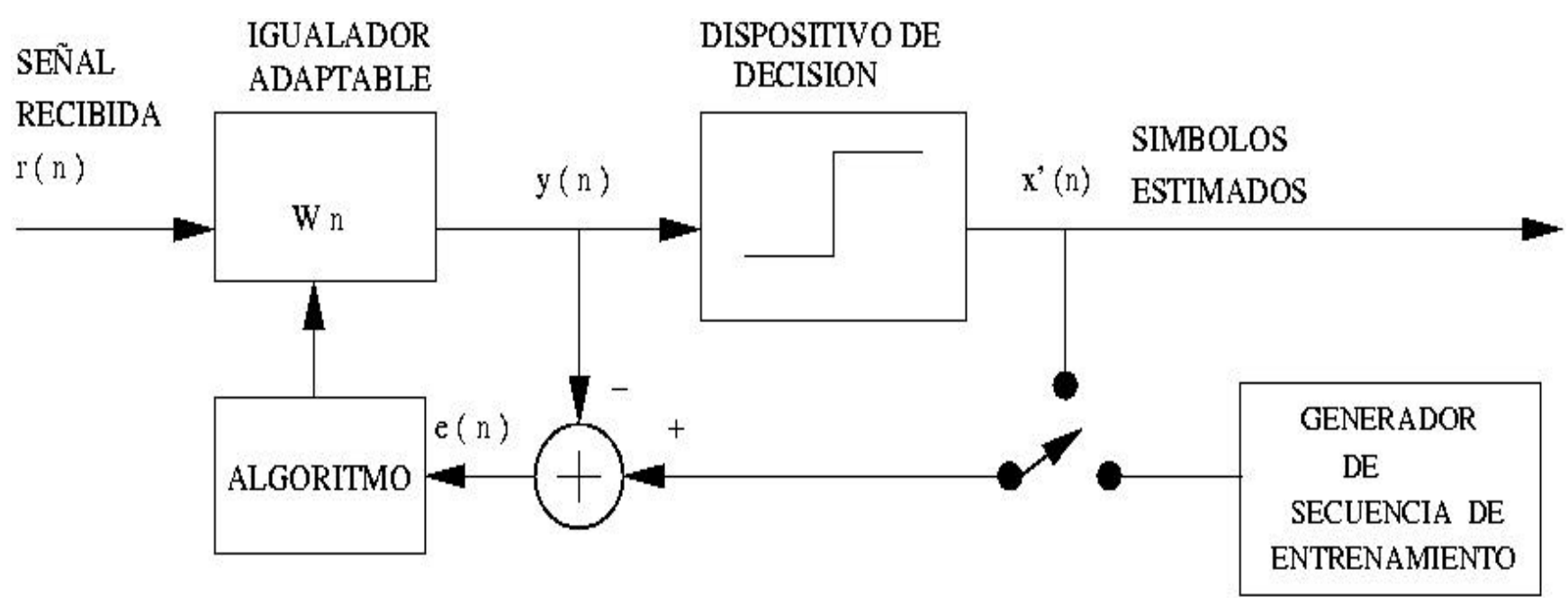

Figura 11. Igualador adaptable con secuencia de entrenamiento 


\section{L.H. Escobar-Salguero}

Los resultados se muestran a continuación, en la figura 12 se tiene la deconvolución del canal $h(n)$ que pro duce una g(n), para verificar que efectivamente $g(n)$ ha deconvolucionado correctamente a $h(n)$, se observa la repre- sentación espectral de $H(z)$ y $G(z)$ en la figura 13 , lo que muestra claramente que estas funciones de transferencia son inversas y resuelven el problema planteado inicialmente.
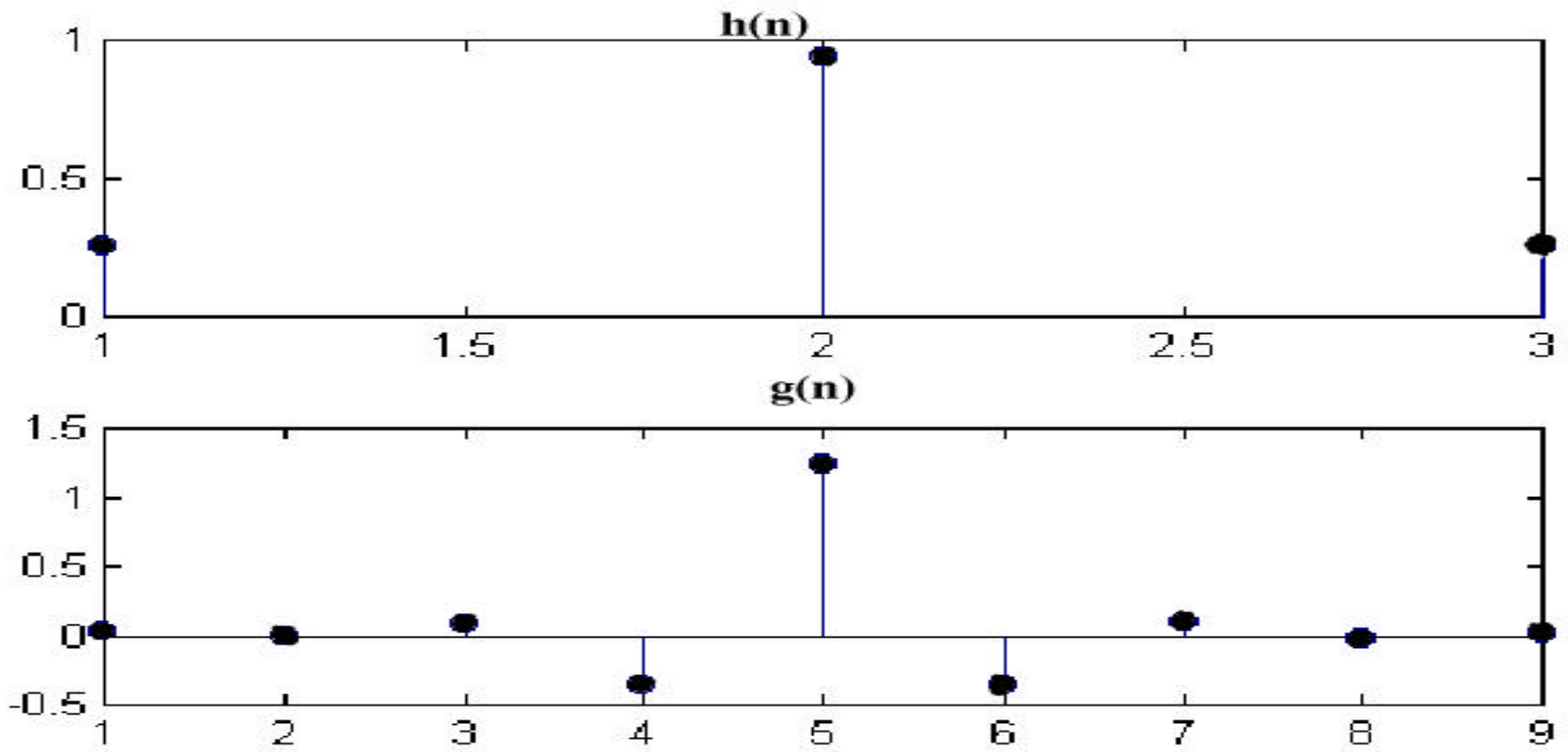

Figura 12. En el tiempo $g(n)$ es la deconvolución de $h(n)$

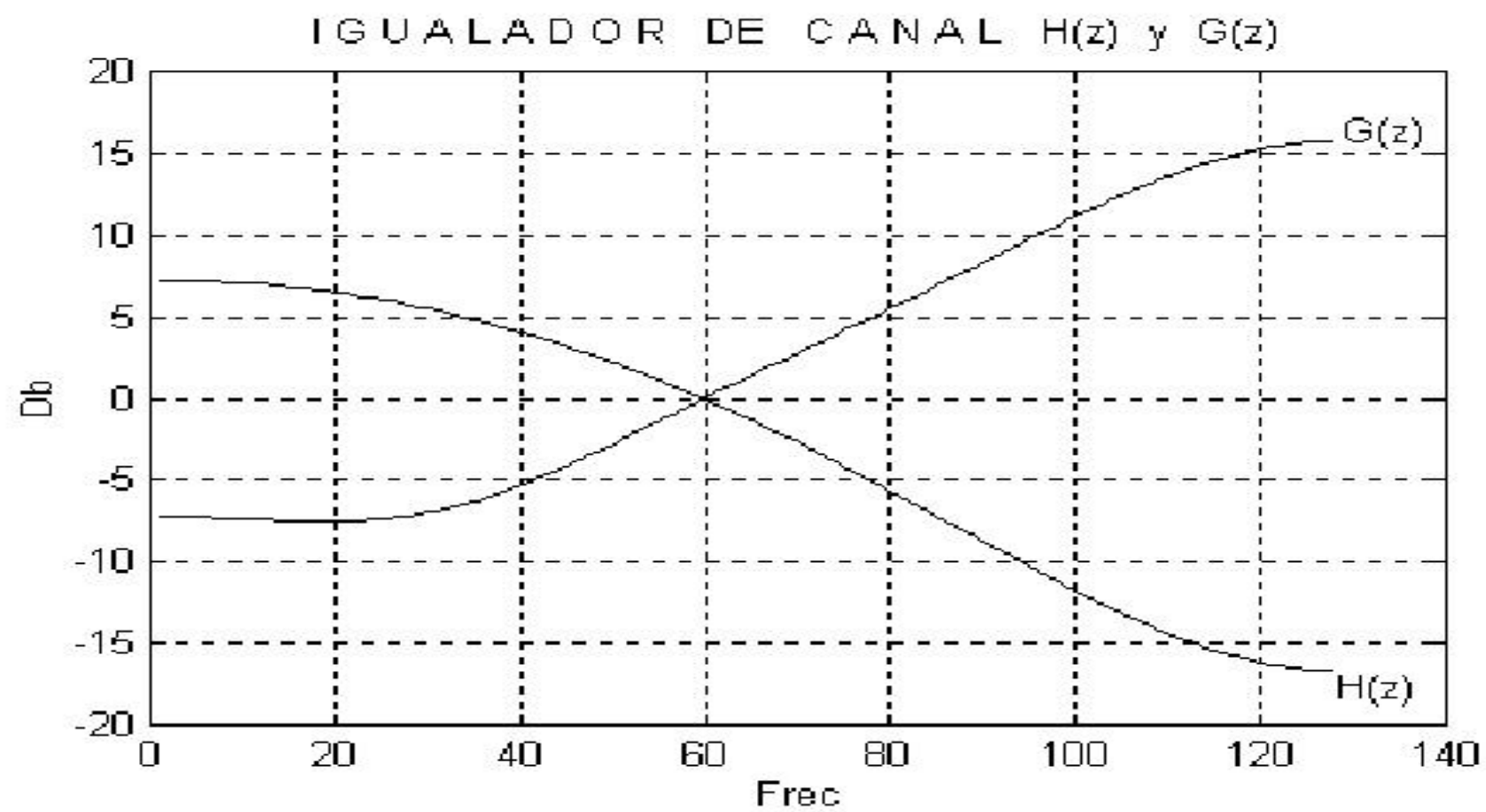

Figura 13. En el espectro $G(z)$ es la inversa de $H(z)$ 


\section{Conclusiones}

Se ha mostrado que los filtros adaptables comparados con los filtros fijos presentan un mejor desempeño cuando se desconocen las características de la señal de entrada o sus estadísticas varían en el tiempo.

La desventaja en el uso del algoritmo LMS es su velocidad de convergencia, así como su sensibilidad al mal acondicionamiento de la matriz R. Por otro lado, los algoritmos rápidos RLS son veloces en converger pero presentan problemas de inestabilidad y su implantación en lenguaje ensamblador necesita muchas operaciones. La mayoría de los algoritmos rápidos que trabajan en estructuras transversales son derivados del algoritmo rápido FRLS, éstos difieren esencialmente de la forma en que se establecen los cálculos de las vari ables intermedias.

La convergencia de los algoritmos está ligada a las condiciones iniciales, la sensibilidad de los errores de cálculo y a la longitud de palabra del sistema digital donde se ejecuten los algoritmos (Alcántara y Escobar, 1998, 1999) y (Escobar, 2000).

La utilización de los algo ritmos de filtrado adaptable depende de la aplicación particular, es decir, si se dispone de mucho tiempo para operar o si los tiempos son cortos se necesitan algoritmos que sean rápidos. El algoritmo LMS puede ser una solución sencilla cuando se trabaja con señales que cambian muy lenta mente con el tiempo.

Se ha mostrado la implantación de algoritmos AFAs, su desempeño y problemáticas, en la solución de problemas reales tales como la predicción, en la cancelación de ruido, la cancelación adaptable de interferencia de $60 \mathrm{hz}$ en una señal de ECG se comporta similar a un filtro supresor de ancho de banda angosta, la cancelación de eco en una línea telefónica y la igualación de canal.

\section{Referencias}

Alcántara R. (1986). Implantation D'algorithmes Rapides Sur des Processeur de Traitement du Signal. These de Docteur Ingenieur, ENST Paris, France.

Carayannis G., Manolakis D. y Kaloupsidis N. (1983). A Fast Sequential Algorithm for Least-Squares Filterings and Prediction. IEEE Trans. on A coustics, 5 peech and Signal Processing. Vol. ASSP 31, No. 6,1394-1402.

Cioffi J.M. (1984). The Fast Transversal Filters for Communications Applications. Ph. D. Dissertation Thesis of Stanffor University.

Alcántara R. y Escobar S.L. (1998). Dynamic Range and Scaling Evaluation in Adap tive Filtering Algo rith ms for DSP Fixed-point Implementation. International Symposium on Information Theory and its Applications ISITA98. México DF, october.

Alcántara R. y Escobar L. (1999). A Comparative TMS DSP Fixed-Point Implementation of FRLS Adaptive Filtering Algorithms Family. The International Conference on Signal Processing Application and Technology and DSP World Workshops, ICSPAT99. Orlando, Florida USA, november.

Escobar S.L. (1997). Algoritmos de filtrado adaptable: Implementación, evaluación, comparación y aplicaciones en telecomunicaciones. Tesis de Maestría en Ingeniería Eléctrica, UNAM, México DF, noviembre. 
Escobar S.L. y Alcántara R. (2000). Fixed Point Arithmetic Using Digital Signal Processors. International Conference on Telecommunications, ICT2000, Acapulco, Guerrero, México, mayo.

Falconer y L. Ljung. (1978). Applica tion of Fast Kalman Estimation to Adaptive Equalization. IEEE Trans., Comm., Vol. COM-26, No. 10, 1439-1446.

Haykin S. (1989). Modern Filters. MacMillan, New York.

Haykin S. (1991). Adaptive Filter Theory. Englewood Cliffs, NJ Prentice-Hall.

Haykin S. (1994). Blind D econvolution. Prentice Hall, Englewood Cliffs, New York.

Messerschmitt D., Hedberg D., Cole C., Haoui A. y Winship P. (1989). Digital Voice Echo Canceller with a TMS32020. Texas instru ments.

Proakis J.G. y Manolakis (1992). Digital Signal Processing, Principles, Algorithms and Applications. Macmillan, Singapure.

Proakis J.G. (1995). Digital Communication. MacGraw-Hill, USA.

Sibul L. (1987). Adaptive Signal Processing. IEEE press. USA.

Treichler J.R., Larimore M.G. y J.C. (1998). Harp. Practical Blind Demodulator for High-Order QAM Signals. Proceedings of IEEE, Vol. 86, No. 10.

Widrow y Stearns. (1985). Adaptive Signal Processing. Prentice-Hall, New Jersey.

\section{Bibliografía sugerida}

Keem S. (1997). Fixed-point Analysis and Simulation of Digital Signal Processing Algorithms. Ph.D Dissertation, Seoul National Univer sity.

Kaloupsidis N. y Theodoridis S. (1993). Adaptive System Identification and Signal
Processing Algorithms. Prentice-Hall, Cambridge G. B..

Long G. y Ling F. (1990). A New Complex System Identification Method and its Application to Echo Canceller Fast Initialization. In Proc. IEEE Int. Conf. Acoust., Speech, Signal Processing, Albu querque, NM ., 1671-1674.

Oppenheim A.V., Schafer R.W. y Buck J.R. (1999). Discrete Time Signal Processing. Prentice-Hall, USA.

Proakis J., Lin R.C. y Nikias C. (1992). Advanced Digital Signal Processing. Macmillan-Maxwell, Ontario Canada.

Treichler J.R., Fijalkow I. y Johnson C.R. (1996). Fractional Spaced Equalizers. IEEE Signal Processing M aga zine, mayo. Ungerboeck G. (1972). Theory on the Speed of Convergence in Adaptive Equalizers for Digital Communication. IBM J. Res. Develop. November.

Webb W.T. y Hanzo L. (1994). Modern Quadrature Amplitude Modulation, Principles and Applications for Fixed and Wireless Channels. Pentech Press \& IEEE Press. London G.B. 
Estudio comparativo y aplicaciones de algoritmos de filtrado adapt able

\section{Semblanza del autor}

Larry Hipólito Escobar-Salguero. Obtuvo el título de ingeniero mecánico electricista en 1992 y de maestro en ingeniería eléctrica con mención honorífica en 1997, ambos en la Facultad de Ingeniería de la UNAM. Actualmente es profesor en la misma institución, ha impartido materias tanto a nivel licenciatura como en posgrado. Es autor y coautor de 10 artículos de congresos nacionales e internacionales y de cinco libros de apuntes publicados por la Facultad de Ingeniería, UNAM. Ha impartido cursos de actualización académica en otras universidades y centros de investigación y ha participado como corresponsable en varios proyectos de investigación patrocinados por PAPIIT, DGAPA. La Facultad de Ingeniería, UNAM le otorgó dos cátedras especiales en los años 2001 y 2002. 